\title{
A Study on Gamification Effectiveness
}

\author{
Petar Cvetkovic ${ }^{1}$, Charly Harbord ${ }^{2} \mathbb{1}^{\mathrm{a}}$ and Helmut Hlavacs ${ }^{1} \mathbb{1}^{\mathrm{b}}$ \\ ${ }^{1}$ University of Vienna, Faculty of Computer Science, Vienna, Austria \\ ${ }^{2}$ Abertay University, School of Design and Informatics, Faculty of Games and Arts, Dundee, Scotland
}

Keywords: Gamification, Effectiveness, Education, Feedback.

\begin{abstract}
Studies have shown that gamification increases motivation and user experience when it comes to a certain behavior or completing a process. Gamification is often deeply associated with naïve animations and stylized text. This paper addresses the effect that visual representation has on the motivation of a subject by measuring their motivation after completing a mundane process, with both entertaining gamification elements as well as gamification elements presented in plain text. For the purposes of this study a within subject design was used to gather data. Participants completed the same mundane task three times, once without any gamification elements, once with pragmatic feedback and once with entertaining feedback. After completion, the participants filled out the same Likert scale survey. The results, evaluated using the Wilcoxon Signed-Ranks test method, indicated that there was not a significant difference in user motivation between the visually stylized and plain text feedback. If conducted on a larger scale, this discovery could lead to a reduction in both time and cost for gamification development.
\end{abstract}

\section{INTRODUCTION}

Gamification is the use of game design elements in non-game settings to engage participants and encourage desired behaviors (Deterding et al., 2011) The premise is that "to create an effect in non-game fields by applying the game mechanics and game thinking which make games fun; for example points, levelup, ranking, achievements, competition, and rewards" (Park and Bae, 2014). Gamification elements have been used broadly in many different branches with the goal to make processes more entertaining and easier to grasp (Deterding et al., 2011). However, it is worth noting that "entertaining" motivational tricks are subjective and are very much dependent on the user's perspective (Kuo and Chuang, 2016). In order to motivate users; gamification inserts game mechanics and dynamics into non-game contexts (de Marcos et al., 2014). Within the field of motivation, they can be seen to be two main branches: extrinsic and intrinsic. Extrinsic motivation refers to an action being completed with the knowledge that there will be some defined reward at the end. Intrinsic motivation however, refers to an action that is completed purely

a (iD) https://orcid.org/0000-0001-7079-852X

b (D) https://orcid.org/0000-0001-6837-674X for the joy of that action. It is this combination of the intrinsic motivation of gameplay and educational activities, which would normally be associated with extrinsic motivation, that is of great interest to both researchers and educators. Additionally, gamification by way of increasing intrinsic motivation also has the potential to improve engagement via the medium of flow (Hamari, 2013; Huotari and Hamari, 2012; Csikszentmihalyi, 2009; Deci and Ryan, 1985). The research described in this paper was designed as a case study to investigate whether the visual presentation of feedback has influence on the intrinsic motivation when completing a process. The hypothesis being: "Does visual representation improve user motivation or just improve the over-all impression of the task?" If the user motivation does not change drastically, then the development time and cost of gamification elements would therefore be reduced. At present, within the gamifiaction process a large proportion of time is devoted to the design of visual elements. The efficacy of gamification was measured by conducting a within subject study with 10 participants and then evaluating the results using the Wilcoxon Signed Ranks Test method.

This paper details the results of the experiment carried out at the University of Vienna in 2019. The remainder of the paper is organized as follows: Sec- 
tion 2 provides information about studies which prove that gamification overall improves the user motivation; Section 3 provides a description of the experiment; Section 4 shares the results of findings; Section 5 shows a detailed interpretation of the results and Section 6 summarizes the findings and discusses future work.

\section{RELATED WORK}

There have been an increasing number of scientific papers that have described the analysis and application of gamification and benefits from it. There has been heavy focus on measuring and showing how gamification elements improve user motivation and according to (Vesa, 2017) more than 50 percent of the organizations who manage an innovative process will gamify their business. The subsections below serve as examples for the application of gamification in different professional situations.

\subsection{Gamification in Education}

Gamification plays a huge role in education. Games, in any form, increase motivation through engagement, participation, loyalty and competition (Alsawaier, 2018). The premise being that by employing the 'entertaining' aspects of games and gameplay to a ordinary educational activities the student motivation to complete those actions should increase (Kuo and Chuang, 2016). According to Chou (Chou, 2019): "...gamification is one of the methods that motivates students to actively participate in class, learn through entertainment by playing with their colleagues and emphasize on the positive competition and also, to make learning fun". Gamification of within the field education is often implemented within the classroom setting during class time. One benefit of within class use is that the students are able to follow their own progress and achievements via instantaneous feedback allowing for an increase in motivation and engagement (Kapp, 2012; Simoes et al., 2013)

\subsection{Gamification in Medicine}

Gamification is breaking the barriers of medicine by using elements to increase motivation to become a blood donor. "...development of applications that can facilitate users to motivate each other doing voluntarily and routinely blood donors, by gamification concept." (Prasetiantowibowo and Lusi-Ani, 2017).

\subsection{Gamification in Sport}

Gamification also influences the world of sports. As stated in Chou (Chou, 2019): "people find it difficult to get time and interest for doing sports activities in the form of recreational sport...gamification principles are used to enhance the experience in those activities." A good example of health and gamification combined is the "Nike+ App". This is a system that calculates and transmits distance run and calories burned of a person by the utilization of a specially designed sensor attached to the running shoe that syncs data with an iPod. By default, it promotes social fun when used with friends, and aids the user to plan, share, and complete exercise plans through an app (Koivisto and Hamari, 2014). Feeding back to the question that the paper aims answers of whether or not the visual presentation of feedback elements matter. Would the "Nike+ App" users run the same distance if the feedback was presented in plain text, or do the animation and pleasing visual design have a motivational effect on performance?

\section{DESCRIPTION OF THE EXPERIMENT}

The purpose of the research study was to explore the impact of the representation of a single gamification element - for the purposes of this study the gamification element used was gamified feedback - on the user's intrinsic motivation and excitement in completing a mundane process. To conduct the study, a mundane process was created and remained constant on the left side of the screen, while the different feedback types changed on the right side.

\subsection{Hypotheses}

- Null hypotheses: The type of feedback has no influence on the intrinsic motivation of completing a process.

- Alternate hypotheses 1: Showing only facts without gamification elements motivate completing the process less than using gamified feedback.

- Alternate hypotheses 2: Using a pragmatic gamified feedback motivates completing the process less (or more) than using entertaining feedback.

\subsection{Experimental Participants}

The study was conducted on June/July 2019. There were no specific categories. The participants were 
taken from the general public. There were no restrictions to who the participants should be, the more diverse, the better. Among the participants, 6 out of 10 were female and 4 out of 10 were male. The average age of the respondents were 22 years old.

\subsection{Game Development}

In order to develop the counting game (described in the following section) and evaluate the experimental results we used a number of software tools. "Balsamiq Mockups" ${ }^{1}$ was used to draw the process and feedback mock-ups during the game design. A screen shot can be seen in Figure 1.

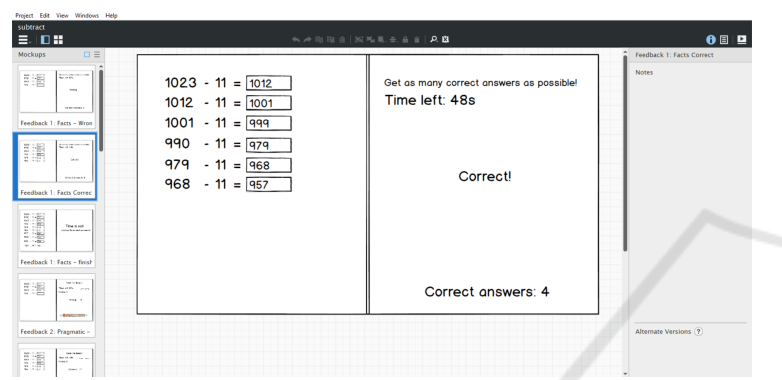

Figure 1: Screenshot of Balsamiq Mockups.

The game was developed using the popular game engine "Unity $3 D^{\prime \prime 2}$. Unity 3D is a general purpose game engine which allows the launch of games on a multitude of platforms, in our case the chosen platform was Windows, a screen shot is shown in Figure 2 .

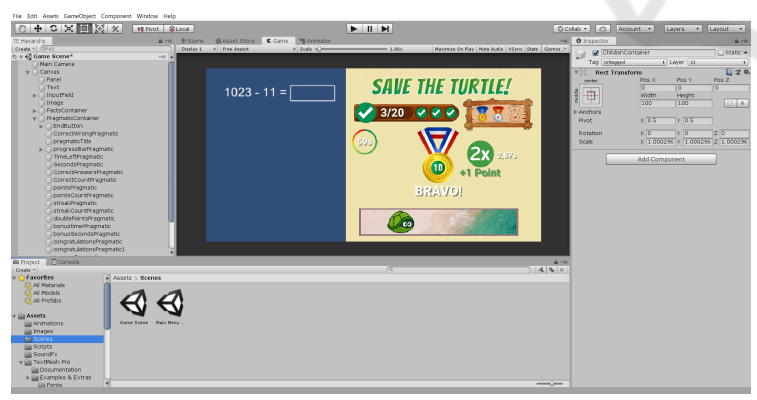

Figure 2: Screenshot of Unity.

Finally, "IBM SPSS Statistics" 3 has been used to derive statistical results.

\footnotetext{
${ }^{1}$ https://balsamiq.com/

${ }^{2}$ https://unity.com/

${ }^{3}$ https://www.ibm.com/analytics/spss-statisticssoftware
}

\subsection{The Counting Game}

For the purposes of the investigation, a mundane process with three different variations of feedback was developed and a questionnaire was designed. In the game, the screen is split in the middle. The left side displayed the mundane process and stayed constant throughout. The right-side displayed the feedback and changed depending on the feedback type. The mundane process was defined to be subtracting the number 11 from another given start number, as many times as possible within 60 seconds. It was clear that such a task soon becomes tedious and is generally considered not to be fun. The counting game offered three levels, each of them representing a different feedback type. Figure 3 shows the game's start menu. At this point, the player can select one of three levels of the game, each level representing one of the three possible feedback types. In the following each of the feedback types is described.

\section{SAVE THE TURTLE!}

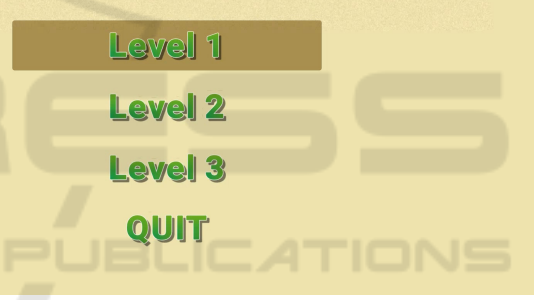

Figure 3: Main menu screen.

\subsubsection{Level 1: No Feedback}

There is no main goal or narrative of the game in this level, the subject merely needed to answer as many questions correctly as they could within 60 seconds. The right-side of the screen shows a countdown timer indicating the remaining time, correct answer count and the correct/wrong text. Also, the decision was made to not use sound effects. Figure 4 shows the intro scene explaining how to play the game for the no-feedback level. Figure 5 shows how the game progresses in the no-feedback level. The left upper image, shows how the game starts, to the right, one answer has been answered correctly. On the left lower image, the game is shown after a number of correct answers. On the lower right image, the case of a wrong answer is shown. 


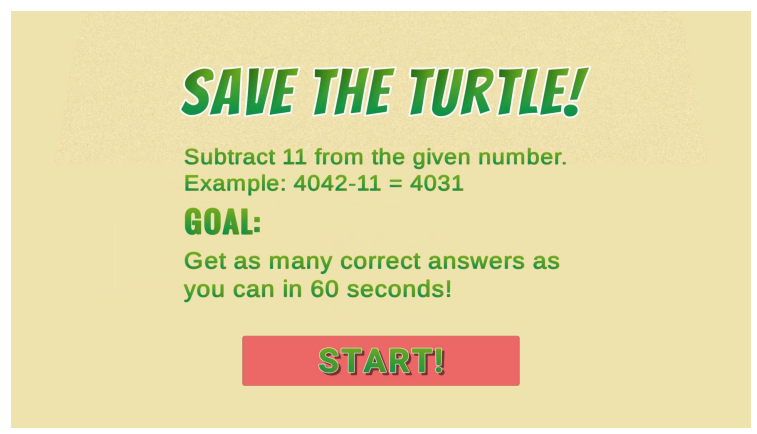

Figure 4: Intro scene no feedback.

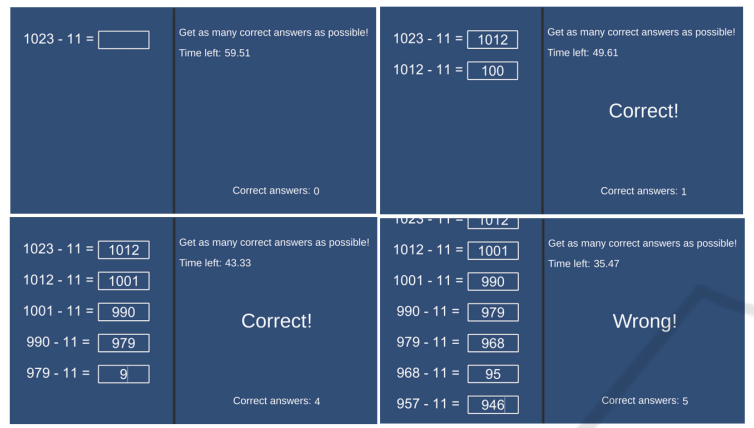

Figure 5: Scenes from no feedback.

\subsubsection{Level 2: Pragmatic Feedback}

This level includes the introduction of a goal: to save a turtle by leading it to the safety of the sea. The goal can be achieved by reaching a total of 20 points. Every correct answer counts as one point, and every wrong answer reduces the score by a point. Depending on the amount of points, the turtle moves closer to or further away from the sea, which is also graphically shown on the right feedback screen. After three correct answers in a row, a double points bonus is awarded. However, the bonus disappears if the user does not submit another correct answer within 4 seconds, or if the next answer is wrong. The feedback is presented as plain text. There are also added sound effects for the correct and wrong answers.

Due to the narrative, this level is considered to be gamified, though only to a bare minimum. Depictions of successes or failures are not exaggerated. Instead, the additional motivation is given mainly due to the narrative, something that gives meaning to the process. Figure 6 shows the intro scene of the game for pragmatic feedback, describing the game functions. Figures 7 and 8 show a progression of this level. Figure 7 depicts the feedback of correct answers, while Figure 8 shows the conquences of submitting wrong answers or even failing to achieve the level goals.

\section{SAVE THE TURTLE!}

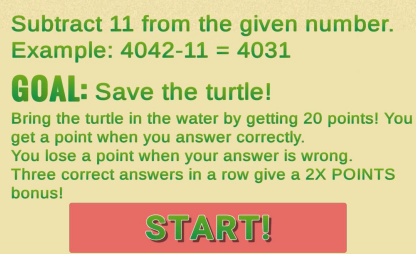

Figure 6: Intro Scene from pragmatic and entertaining feedback.

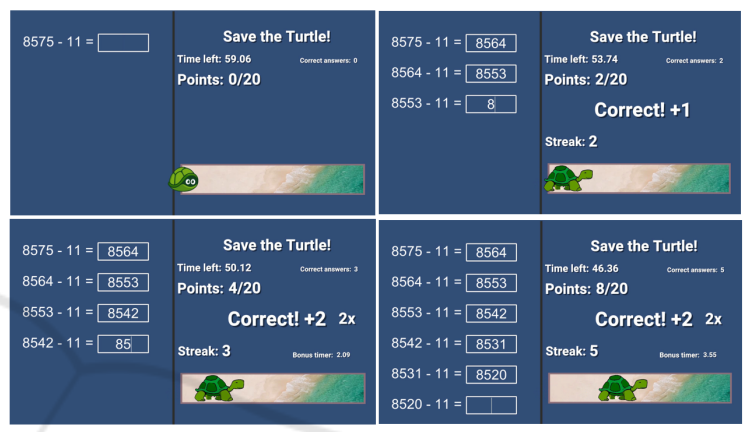

Figure 7: Scenes from pragmatic feedback.

\subsubsection{Level 3: Entertaining Feedback}

The goal and mechanics of this level are kept the same as the pragmatic feedback level. However, the feedback differs in two ways: firstly, it utilizes colorful animation, and more sound effects. Secondly, the creation of tangible achievements in the form of medals which can be gained by getting three, five and ten correct answers in a row. Sound effects are added for typing, the correct answer, the wrong answer, bonus, bonus loss, medals, and general background music. The introduction to the 'game' was kept the same as pragmatic feedback (Huotari and Hamari, 2012), as depicted in Figure 6.

This level is considered to be strongly gamified, it looks and feels like a computer game made for entertainment purposes only. The gamification of feedback here is exaggerated, and represents an extreme "sugar coating" of the mundane counting process. Figure [9] shows the feedback of correct answers, providing

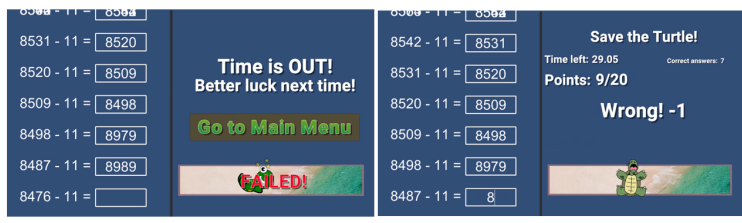

Figure 8: Scenes from pragmatic feedback. 
multiple positive reactions. Figure 10 on the upper row shows the feeback for wrong answers or failure, the rows below show feedback for success including streaks and medals.
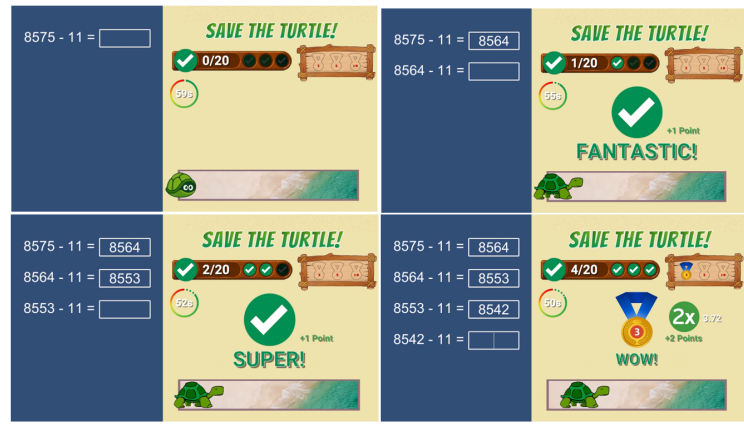

Figure 9: Scenes from childish feedback.
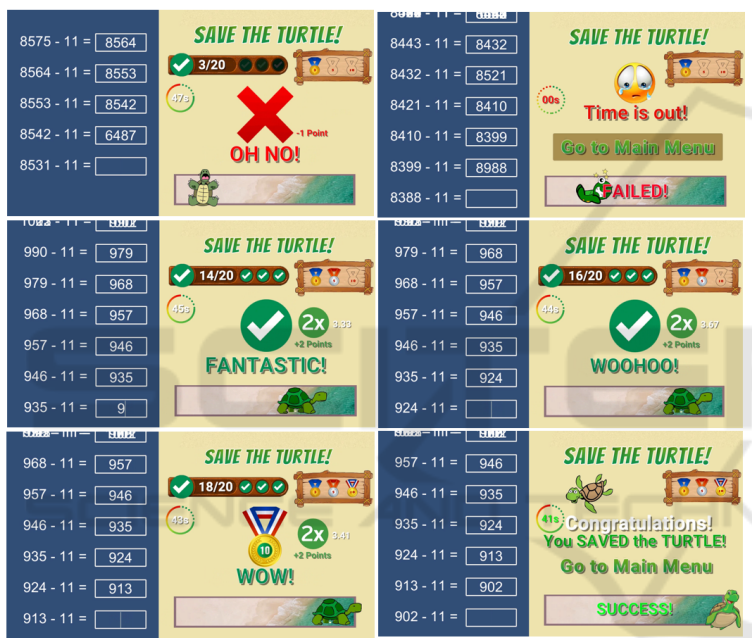

Figure 10: Scenes from childish feedback.

\section{EXPERIMENTAL EVALUATION}

Data was collected in person and there were no special conditions required for running the project. A within subject study design was used as this allowed for the same users to test all conditions, complete the process three times and fill out the same questionnaire after each completion. The feedback order was randomized for preventing any particular ordering of introducing a bias to the results. The survey answers were rated with a Likert scale utilizing 5 levels: strongly disagree, weakly disagree, neutral, weakly agree, and strongly agree. Values between -2 (strongly disagree) to +2 (strongly agree) were awarded. The survey questions are due (Deterding et al., 2011) and formulated in a positive way. They can be found in the Table 1 .
Table 1: List of questions from the survey.

\begin{tabular}{|l|l|}
\hline & Please rate the following statements. \\
\hline 1 & Completing the process was fun \\
\hline 2 & I felt excited \\
\hline 3 & I never felt bored \\
\hline 4 & I would complete the process again \\
\hline 5 & I felt like a MATH GENIUS \\
\hline 6 & I felt like I was really saving the turtle \\
\hline 7 & $\begin{array}{l}\text { I felt motivated to get as many correct } \\
\text { answers as possible }\end{array}$ \\
\hline 8 & Answering correctly felt rewarding \\
\hline 9 & $\begin{array}{l}\text { I double checked my answer to make } \\
\text { sure I don't answer wrong }\end{array}$ \\
\hline 10 & I forgot I was doing math \\
\hline
\end{tabular}

\subsection{Experimental Results}

A Wilcoxon signed-ranks test were used for result evaluation, because the within subject design demands that the same subjects test multiple times, and it results in paired samples. The Wilcoxon signedrank test is a non-parametric hypothesis test deciding whether two related samples stem from the same population or not (Wilcoxon, 1945). Data comes in pairs, and the null-hypothesis is that they come from the same population. Each pair is chosen randomly and independently,

To test the hypotheses, the Wilcoxon signed ranks test was executed three times. First the "No feedback" and "Pragmatic feedback" results were compared. Followed by "no feedback" and "Entertaining feedback", and finally "Pragmatic feedback" and "Entertaining feedback". The survey consists of 10 questions; for each question, the sum of values from all participants was calculated, which resulted in 10 specific values - one value for each question. So, the sum of all participants ratings for one answer was viewed as one score. This in return gives 10 answers for each feedback type, and the difference between them are compared. Results were calculated using the SPSS statistics tool. Significance level alpha for a Wilcoxon signed ranks test was 0.05 .

In order for there to be a significant difference between two median values, the $p$ value has to be less than significance level value, and if it is, the null hypothesis which states that there is no difference is rejected. The total scores that were calculated are shown in Table 2, (Park and Bae, 2014).

Though for a Wilcoxon singed-ranks test an interval scale is recommended, for within-pair comparisons an ordinal scale is sufficient. We can thus submit the data from Table 2 to this test. 
Table 2: Sum of scores for each question of the survey.

\begin{tabular}{|c|c|c|c|}
\hline Question & No feedb & Pragmatic & Entertaining \\
\hline 1 & 2 & 6 & 8 \\
\hline 2 & 1 & 12 & 8 \\
\hline 3 & 3 & 10 & 9 \\
\hline 4 & -5 & 6 & 4 \\
\hline 5 & -4 & -5 & -3 \\
\hline 6 & -11 & -11 & -1 \\
\hline 7 & 10 & 16 & 12 \\
\hline 8 & -2 & 13 & 10 \\
\hline 9 & -11 & -11 & -10 \\
\hline 10 & -6 & -6 & -7 \\
\hline
\end{tabular}

\subsection{No Feedback vs. Pragmatic Feedback}

In this section we compare the results from "No feedback" to those of "Pragmatic feedback". The null hypothesis and its alternative are given by the following statements:

Null Hypothesis: The type of feedback has no influence on the intrinsic motivation of completing a process.

Alternative 1: Only facts without gamification elements motivate completing the process less than gamified feedback. (the type of feedback HAS influence on intrinsic motivation of completing a process)

Looking at the descriptive statistics shown in Table 3 , we can see that the mean value of all answers for "No feedback" is negative (-2.30) and positive (3.00) for the "Pragmatic feedback", which already shows that there is a difference between the two feedback types.

Table 3: Descriptive Statistics for "No feedback" and "Pragmatic feedback", including 25th, 50th (median), and 75th percentiles.

\begin{tabular}{|l|c|c|c|c|c|c|c|c|}
\hline \multicolumn{10}{|c|}{ Descriptive statistics } \\
\hline & N & Mean & SDev & Min & Max & 25 th & 50 th & 75 th \\
\hline No fb & 10 & -2.30 & 6.533 & -11 & 10 & -7.25 & -3.00 & 2.25 \\
\hline Pragmatic & 10 & 3.00 & 10.296 & -11 & 16 & -7.25 & 6.00 & 12.25 \\
\hline
\end{tabular}

If we pay attention to the ranks shown in Table 4, we notice that there is only one negative rank, meaning only one from ten questions was in favor of "No feedback", six of them were in favor of "Pragmatic feedback" and three of them were tied.

Finally looking at the actual test statistics as depicted in Table 5, we notice that the $Z$ value is -2.201 and the $p$ value is 0.028 .

As already stated, if the $p$ value is smaller than the alpha value, we reject the null hypothesis. In our case 0.028 is in fact smaller than 0.05 which means that the type of feedback HAS influcence on the intrinsic
Table 4: Ranks table for "No feedback" and "Pragmatic feedback".

\begin{tabular}{|l|l|c|c|c|}
\hline \multicolumn{5}{|c|}{ Ranks } \\
\hline \multicolumn{2}{|c|}{} & N & $\begin{array}{c}\text { Mean } \\
\text { Rank }\end{array}$ & $\begin{array}{c}\text { Sum of } \\
\text { Ranks }\end{array}$ \\
\hline \multirow{4}{*}{$\begin{array}{l}\text { Pragm. fb- } \\
\text { No fb }\end{array}$} & Neg. Ranks & $1^{a}$ & 1.00 & 1.00 \\
\cline { 2 - 5 } & Pos. Ranks & $6^{b}$ & 4.50 & 27.00 \\
\cline { 2 - 5 } & Ties & $3^{c}$ & & \\
\cline { 2 - 5 } & Total & 10 & & \\
\hline
\end{tabular}

a. Pragmatic $\mathrm{fb}<\mathrm{No} \mathrm{fb}$

b. Pragmatic $\mathrm{fb}>\mathrm{No} \mathrm{fb}$

c. Pragmatic $\mathrm{fb}=\mathrm{No} \mathrm{fb}$

Table 5: Test statistics for "No feedback" and "Pragmatic feedback".

\begin{tabular}{|l|c|}
\hline \multicolumn{2}{|c|}{ Test Statistics $^{a}$} \\
\hline & $\begin{array}{c}\text { Pragmatic fb } \\
- \text { No fb }\end{array}$ \\
\hline Z & $-2.201^{b}$ \\
\hline Asymp. Sig. (2-Tailed) & 0.028 \\
\hline
\end{tabular}

a. Wilcoxon Signed Ranks Test

b. Based on negative ranks.

motivation of completing a process. So we reject the null hypothesis in favor of the alternative hypothesis.

We can conclude that a Wilcoxon Signed-Ranks test indicated that only facts without gamification elements motivate completing the process less than gamified feedback. Thus the type of feedback HAS influence on the intrinsic motivation of completing a process.

To take this a step further, we can calculate the effect size by using the following formula:

$$
r=\left|\frac{Z}{\sqrt{N}}\right| .
$$

The formula includes the $Z$ value and $N$ representing the number of samples which is 10 for each group, which means 20.

$$
r=\left|\frac{(-2.201)}{\sqrt{20}}\right|=0.4921 .
$$

Based on the following effect size interpretations as shown in Table 6 we can conclude that the effect size is medium but very close to being large.

Table 6: Cohen's effect size
\begin{tabular}{|c|c|}
\hline Effect size & $\mathrm{r}$ \\
\hline Small & 0.10 \\
\hline Medium & 0.30 \\
\hline Large & 0.50 \\
\hline
\end{tabular}

We conclude that the participants enjoyed the pragmatic feedback more than no feedback. Finally, 
the hypothesis test summary from SPSS statistics tool is shown in Table 7, including the decision about the null hypothesis.

Table 7: Hypothesis test summary for "No feedback" vs. "Pragmatic feedback".

\begin{tabular}{|l|l|c|l|}
\hline \multicolumn{4}{|c|}{ Hypothesis Test Summary } \\
\hline Null Hyp & Test & Sig. & Decision \\
\hline Median of & Related-Samples & 0.028 & Reject \\
differences btw & Wilcoxon & & null \\
No fb and & Signed Rank & & hypoth. \\
Pragm. fb is 0 & Test & & \\
\hline
\end{tabular}

The significance level is 0.05 .

\subsection{No Feedback vs. Entertaining Feedback}

There was no real need to test the "No feedback" with "Entertaining feedback" but for checking consistency it was also carried out, as it might give some interesting insight. The hypothesis remains the same:

Null Hypothesis: The type of feedback has no influence on the intrinsic motivation of completing a process.

Alternative 1: Only facts without gamification elements motivate completing the process less than gamified feedback. (the type of feedback HAS influence on intrinsic motivation of completing a process)

The mean value of all answers for "No feedback" is the same as before: negative $(-2.30)$ and we can see that the "Entertaining feedback" has the same mean value (see Table 8) as the "Pragmatic feedback": positive (3.00) for the "Pragmatic feedback", which makes it interesting for the comparison between "Pragmatic" and "Entertaining" feedback.

Table 8: Descriptive Statistics for "No feedback" vs. "Entertaining feedback".

\begin{tabular}{|l|c|c|c|c|c|c|c|c|}
\hline \multicolumn{10}{|c|}{ Descriptive statistics } \\
\hline & N & Mean & SDev & Min & Max & 25 th & 50 th & 75 th \\
\hline No fb & 10 & -2.30 & 6.533 & -11 & 10 & -7.25 & -3.00 & 2.25 \\
\hline Childish fb & 10 & 3.00 & 7.732 & -10 & 12 & -4.00 & 6.00 & 9.25 \\
\hline
\end{tabular}

The resulting ranks (see Table 9) from "Entertaining feedback" performed a lot better than "Pragmatic feedback" with nine positive and one negative ranks, meaning only one from ten questions was in favor of "No feedback" and all the rest were in favor of "Entertaining feedback".

A conclusion can already be drawn that there is a significant difference between entertaining and no feedback, but to verify, it was noticed that the $Z$ value is -2.608 and the $p$ value is 0.009 , which means that $p$ is much smaller than alpha (0.05) and the null hypothesis is rejected, as shown in Table 10.
Table 9: Ranks table for "No feedback" vs. "Entertaining feedback".

\begin{tabular}{|l|l|c|c|c|}
\hline \multicolumn{5}{|c|}{ Ranks } \\
\hline \multicolumn{2}{|c|}{} & N & $\begin{array}{c}\text { Mean } \\
\text { Rank }\end{array}$ & $\begin{array}{c}\text { Sum of } \\
\text { Ranks }\end{array}$ \\
\hline \multirow{3}{*}{$\begin{array}{l}\text { Childish fb- } \\
\text { No fb }\end{array}$} & Negative Ranks & $1^{a}$ & 2.00 & 2.00 \\
\cline { 2 - 5 } & Positive Ranks & $9^{b}$ & 5.89 & 53.00 \\
\cline { 2 - 5 } & Ties & $0^{c}$ & & \\
\cline { 2 - 5 } & Total & 10 & & \\
\hline
\end{tabular}

a. Entertaining $\mathrm{fb}<\mathrm{No} \mathrm{fb}$

b. Entertaining $\mathrm{fb}>\mathrm{No} \mathrm{fb}$

c. Entertaining $\mathrm{fb}=\mathrm{No} \mathrm{fb}$

Table 10: Test statistics for "No feedback" vs. "Entertaining feedback".

\begin{tabular}{|l|c|}
\hline \multicolumn{2}{|c|}{ Test Statistics $^{a}$} \\
\hline & $\begin{array}{c}\text { Childish fb } \\
- \text { No fb }\end{array}$ \\
\hline Z & $-2.608^{b}$ \\
\hline Asymp. Sig. (2-Tailed) & 0.009 \\
\hline
\end{tabular}

a. Wilcoxon Signed Ranks Test

b. Based on negative ranks.

We can conclude that a Wilcoxon Signed-Ranks test indicated that seeing only facts without gamification elements motivate completing the process less than gamified feedback. Thus the type of feedback HAS influence on the intrinsic motivation of completing a process.

The effect size for "Entertaining feedback" vs. "No feedback" is

$$
r=\left|\frac{(-2.608)}{\sqrt{20}}\right|=0.4721 .
$$

This means that the effect size is medium, but we can also see that the effect size is actually smaller than the one from "Pragmatic feedback", already indicating that going from pragmatic to entertaining might not necessarily result in an increased motivation.

Finally, the hypothesis test summary from SPSS is shown in Table 11.

Table 11: Hypothesis test summary for "No feedback" vs. "Entertaining feedback".

\begin{tabular}{|c|c|c|c|}
\hline \multicolumn{4}{|c|}{ Hypothesis Test Summary } \\
\hline Null Hypothesis & Test & Sig. & Decision \\
\hline $\begin{array}{l}\text { Median of diffs. } \\
\text { between No fb and } \\
\text { Entertaining fb equals } 0\end{array}$ & $\begin{array}{l}\text { Related-Samples } \\
\text { Wilcoxon Signed } \\
\text { Rank Test }\end{array}$ & 0.009 & $\begin{array}{l}\text { Reject } \\
\text { the null } \\
\text { hypothesis }\end{array}$ \\
\hline
\end{tabular}

\subsection{Pragmatic Feedback vs. Entertaining Feedback}

Finally, we compare the two gamification level "Pragmatic feedback" vs. "Entertaining feedback". Null 
hypothesis and alternative are given in the following statements:

Null Hypothesis: "Pragmatic feedback" motivates completing the process the same as "Entertaining feedback".

Alternative 1: "Pragmatic feedback" motivates completing the process less (or more) than "Entertaining feedback".

Both feedback types were compared with "No feedback" and the mean values were the same, but "Entertaining feedback" did a lot better on the ranks, and "Pragmatic feedback" had a bigger effect size. Comparing both results might shed light on the question whether there is a significant difference between the two feedback types according to the participants. As demonstrated previously, when comparing both feedback types to "No feedback", the mean value of all answers for "Pragmatic feedback" and "Entertaining feedback" are the same (3.00), see Table 12. The minimum value of pragmatic was -11 , and -10 for entertaining, and maximum values are 16 and 12 for pragmatic and entertaining respectfully. The descriptive statistics are still ambiguous, which is why it was decided to proceed to the actual Wilcoxon signedranks test results.

Table 12: Descriptive Statistics for "Pragmatic feedback" vs. "Entertaining feedback".

\begin{tabular}{|l|c|c|c|c|c|c|c|c|}
\hline \multicolumn{10}{|c|}{ Descriptive statistics } \\
\hline & N & Mean & SDev & Min & Max & 25 th & 50 th & 75 th \\
\hline Pragmatic fb & 10 & 3.00 & 10.296 & -11 & 16 & -7.25 & 6.00 & 12.25 \\
\hline Entertaining fb & 10 & 3.00 & 7.732 & -10 & 12 & -4.00 & 6.00 & 9.25 \\
\hline
\end{tabular}

From a total of 10 questions, 6 of them were in favor of the pragmatic feedback and 4 of them were in favor of the Entertaining feedback (see Table 13). This is very interesting, because it could be assumed that the inclusion of animation, sound effects and graphical presentation would be more appealing than plain text, but it appears that the pragmatic feedback performed better.

Table 13: Ranks table for "Pragmatic feedback" vs. "Entertaining feedback".

\begin{tabular}{|c|c|c|c|c|}
\hline \multicolumn{5}{|c|}{ Ranks } \\
\hline & & $\mathrm{N}$ & $\begin{array}{l}\text { Mean } \\
\text { Rank }\end{array}$ & $\begin{array}{l}\text { Sum of } \\
\text { Ranks }\end{array}$ \\
\hline \multirow{4}{*}{$\begin{array}{l}\text { Childish fb- } \\
\text { Pragmatic fb }\end{array}$} & Negative Ranks & $6^{a}$ & 5.50 & 33.00 \\
\hline & Positive Ranks & $4^{b}$ & 5.50 & 22.00 \\
\hline & Ties & $0^{c}$ & & \\
\hline & Total & 10 & & \\
\hline
\end{tabular}

Our $Z$ value is -0.564 and the $p$ value is 0.573 as shown in Table 14. 0.573 is bigger than 0.05 which means that we retain the null hypothesis and
Table 14: Test statistics for "Entertaining feedback" vs. "Pragmatic feedback".

\begin{tabular}{|l|c|}
\hline \multicolumn{2}{|c|}{ Test Statistics $^{a}$} \\
\hline & $\begin{array}{c}\text { Childish fb } \\
\text { - Pragmatic fb }\end{array}$ \\
\hline Z & $-0.564^{b}$ \\
\hline Asymp. Sig. (2-Tailed) & 0.573 \\
\hline
\end{tabular}

a. Wilcoxon Signed Ranks Test

b. Based on negative ranks.

that "Pragmatic feedback" motivates completing the process in the same way as "Entertaining feedback". Even more, when considering the ranks we can conclude that the "Pragmatic feedback" actually performed better than the "Entertaining feedback." The effect size is calculated to be

$$
r=\left|\frac{(-0.564)}{\sqrt{20}}\right|=0.1261 .
$$

The effect size $r$ is thus small, close to non-existent. We can conclude that subjects did not experience significant difference in intrinsic motivation for completing the process when it was presented in plain text versus entertaining graphics.

Finally, the hypothesis test summary from SPSS is shown in Table 15.

Table 15: Hypothesis test summary for "Pragmatic feedback" vs. "Entertaining feedback".

\begin{tabular}{|c|c|c|c|}
\hline \multicolumn{4}{|c|}{ Hypothesis Test Summary } \\
\hline Null Hypothesis & Test & Sig. & Decision \\
\hline $\begin{array}{l}\text { Median of diffs. } \\
\text { betw. Pragm. fb and } \\
\text { Entertaining fb equals } 0\end{array}$ & $\begin{array}{l}\text { Related-Samples } \\
\text { Wilcoxon Signed } \\
\text { Rank Test }\end{array}$ & 0.573 & $\begin{array}{l}\text { Retain } \\
\text { the null } \\
\text { hypothesis }\end{array}$ \\
\hline
\end{tabular}

Asymptotic significances are displayed. Significance level is 0.05 .

\section{DISCUSSION}

As previously noted, there are already a number of studies have been conducted showing that a mundane process becomes easier to complete with introduction of gamification elements. The results of this research mirror those that have gone before, with our participants indicating that "No feedback" performs significantly worse than pragmatic or entertaining.

It was intriguing to find out that visual elements do not play a role in motivation. It was previously stated that "Entertaining feedback" would outperform "Pragmatic feedback", but our results have shown that the motivation does not significantly change depending on the visuals. The implementation of "Entertaining feedback" took more than twice the time of the implementation of "Pragmatic feedback" and "No feedback" combined. Since our results show that this ad- 
ditional implementation effort does not improve participant motivation, it makes sense to save this effort in favor of other aspects, like logging more data, or increasing the length of the game.

It is also interesting to note that some participants really focused on the sound effects. "Entertaining feedback" had better sound effects, but pragmatic had a distinct "TRUE" or "FALSE" sound effects which the subjects reported finding really helpful. One of the participants had a notable comment for "No feedback". The participant kept answering incorrectly without noticing, because their attention was not on the visual feedback, but the sound feedback, which is missing in the "No feedback" level. The comment was: "The problem was I did not hear I was wrong!". It can be said that whilst gamified feedback elements are important and improve user motivation, the way that they are presented does not make a drastic difference.

\subsection{Limitations of the Study}

The main limitation of this approach was that the study was the amount of people reached who participated. It was not possible to reach a wide variety of ages, professions etc. For example, it would be interesting to see how an 8-year-old would react to doing math in order to save the turtle.

\section{CONCLUSIONS AND FUTURE WORK}

In this paper, the value of the gamification of feedback was addressed together with the importance of visual presentation. Our main contribution is that users are not significantly more motivated to complete a mundane process when feedback is presented in an entertaining way rather than as plain text.

\section{REFERENCES}

Alsawaier, R. (2018). The effect of gamification on motivation and engagement. International Journal of Information and Learning Technology Vol. 35 No. 1, pages pp. 56-79.

Chou, Y. (2019). Actionable gamification: Beyond points, badges, and leaderboards. Packt Publishing Ltd.

Csikszentmihalyi, M. (2009). Flow. Harper and Row., New York.

de Marcos, L., Domiguez, A., Saenz-de Navarrete, J., and Pages, C. (2014). An empirical study comparing gamification and social networking on e-learning. Computers \& Education 75, pages pp.82-91.
Deci, E. and Ryan, R. (1985). Intrinsic motivation and selfdetermination in human behavior. Plenum, New York.

Deterding, S., Dixon, D., Khaled, R., and Nacke, L. (2011). From game design elements to gamefulness: defining gamification. In 15th International academic MindTrek conference: Envisioning future media environments., pages pp. 9-15.

Hamari, J. (2013). Transforming homo economicus into homo ludens: A field experiment on gamification in a utilitarian peer-to-peer trading service. Electronic Commerce Research and Applications 12(4), pages pp.236-245.

Huotari, K. and Hamari, J. (2012). Defining gamification - a service marketing perspective. In The 16th International Academic MindTrek Conference., pages pp. 17-22., Tampere.

Kapp, K. (2012). The gamification of learning and instruction. Pfeiffer, San Francisco, CA.

Koivisto, J. and Hamari, J. (2014). Demographic differences in perceived benefits from gamification. Computers in Human Behavior 35, pages pp. 179-188.

Kuo, M. and Chuang, T. (2016). How gamification motivates visits and engagement for online academic dissemination: An empirical study. Computers in Human Behavior 55, pages pp. 16-27.

Park, H. and Bae, J. (2014). Study and research of gamification design. Int. J. Softw. Eng. Appl.

Prasetiantowibowo, L. S. N. and Lusi-Ani, C. E. (2017). Implementation of gamification to improve blood donors by peer motivation application.

Simoes, J., Redondo, R., and Vilas, A. (2013). A social gamification framework for a k-6 learning platform. Computers in Human Behavior 29(2), pages pp.345353.

Vesa, M. e. a. (2017). Computer games and organization studies. Organization Studies 38(2), page pp. 273-284.

Wilcoxon, F. (1945). Individual comparisons by ranking methods. Biometrics Bulletin 1 (6), page pp. 80-83. 